\title{
Experimental Demonstration of Spectral Non-reciprocity and Unidirectionality in Volumetric and Printed Magnetic Photonic Crystals
}

\author{
$\underline{\text { Kubilay Sertel }}^{1}$, Lanlin Zhang ${ }^{1}$, Nil Apaydin ${ }^{1}$ and John L. Volakis ${ }^{1}$ \\ ${ }^{1}$ ElectroScience Laboratory, Dept. of Electrical and Computer Engineering, The Ohio State University, \\ 1320 Kinnear Road, Columbus OH 43212, USA \\ \{sertel.1, zhang.471, apaydin.3, volakis.1\}@osu.edu
}

\begin{abstract}
We present experimental confirmation of spectral non-reciprocity and unidirectional propagation in magnetic photonic crystals. The unique dispersion properties of such periodic anisotropic/magnetic media were also emulated on simpler printed structures. We outline two designs that exhibit non-reciprocal dispersion diagrams $($ i.e. $\omega(K) \neq \omega(-K))$ in volumetric and printed magnetic photonic crystals. Due to spectral non-reciprocity, unidirectional propagation properties were experimentally observed. These designs can also be tuned such that a stationary inflection point is achieved within the first propagation band, where $\omega^{\prime}=\omega^{\prime \prime}=\omega^{\prime \prime \prime}=0$ (where $\omega^{\prime}=\partial \omega \partial K$ ). This special mode is referred to as the "frozen mode" (i.e. zero group velocity). Correspondingly, a significantly longer group delay is observed in experiments. These measurements constitute the first experimental demonstration of the unique properties of the subject magnetic photonic crystals.
\end{abstract}

\section{Introduction}

Metamaterials and dispersion engineering have transformed microwave component and antenna designs over the past decade. Their novel properties have allowed for controlled electromagnetic phase velocity [1,2] leading to smaller antennas. Although most metamaterials in the literature are based on textured/periodic isotropic materials (and conductive structures), anisotropy allows for many more degrees of freedom and new dispersion characteristics. In this paper, we focus on magnetic photonic crystals (MPCs) consisting of anisotropic dielectric $(A)$ and ferrimagnetic $(F)$ layers. A unique arrangement of these layers leads to new modes due to spectral non-reciprocity, tunable dispersion and unidirectional wave propagation [3-6]. These new modes are expected to find applications in new antenna and microwave components that can impact a variety of RF devices. Here, we present initial experimental validation of the new modes and phenomena supported by the MPCs.

\section{Volumetric and Printed Magnetic Photonic Crystals}

The volumetric version of MPCs can be realized using low-loss anisotropic dielectric and ferromagnetic layers as shown in Fig. 1(a). The two anisotropic $A$ layers are misaligned, acting as polarization filters. They are placed around the ferromagnetic $F$ layer which introduces Faraday rotation via an external magnetic bias field along the propagation direction [7]. By fine tuning material properties and geometrical dimensions, it is possible to design the dispersion of the volumetric MPC for non-reciprocal propagation and to exhibit a stationary inflection behavior, as depicted in Fig. $1(\mathrm{c})$.

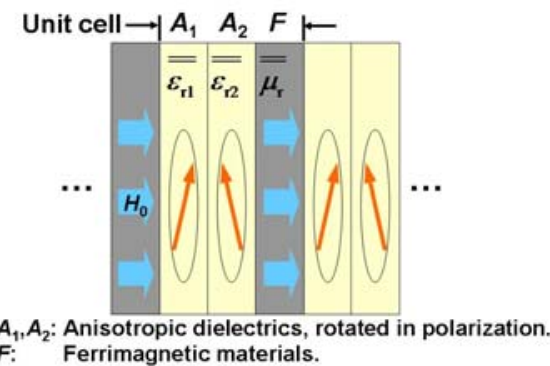

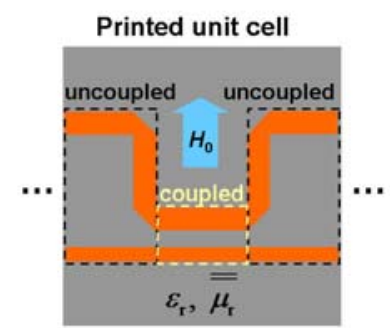

(b)

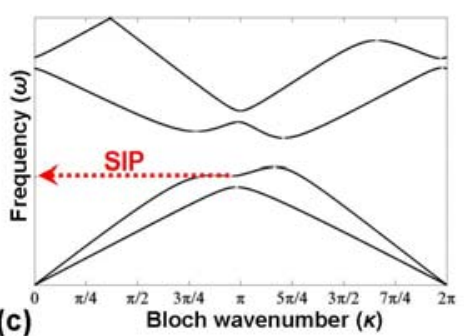

(c)

Fig. 1: (a) Illustration of the MPC layers composed of periodically arranged anisotropic dielectrics and isotropic ferrimagnetic materials. (b) Coupled-lines circuit that emulates the behavior of anisotropic layers (of the MPC) on an isotropic ferrimagnetic substrate (top view). (c) A typical dispersion diagram of an infinite MPC, showing a stationary inflection point (SIP). 
Alternatively, the same dispersion behavior can be achieved on a simple printed structure [8], as depicted in Fig.1(b). The overall dispersion of this unit cell (consisting of a pair of partially-coupled microstrip transmission lines printed on a ferrite substrate) is also non-reciprocal and can be tuned to exhibit the stationary behavior, much like the volumetric MPC.

Below, we describe the fabrication of volumetric and printed MPC prototypes using off-the-shelf materials. In the case of volumetric MPCs, wave propagation along the laminate direction is achieved (see [9])8. By comparison, the printed MPC structure exhibits similar dispersion curves for waves propagating along the transmission line [10]. Experiment data are presented and compared with simulation to validate the proposed MPC properties.

\subsection{Design, Fabrication and Experimental Demonstration of Volumetric MPCs}

The volumetric MPC structure is shown in Fig. 2. A 15 unit-cell structure was constructed using low-loss Cuflon and calcium vanadium garnet for the dielectric and ferromagnetic layers, respectively. To emulate anisotropy, conductive strips were printed on both sides of the Cuflon. All material parameters are given in Table 1 below.
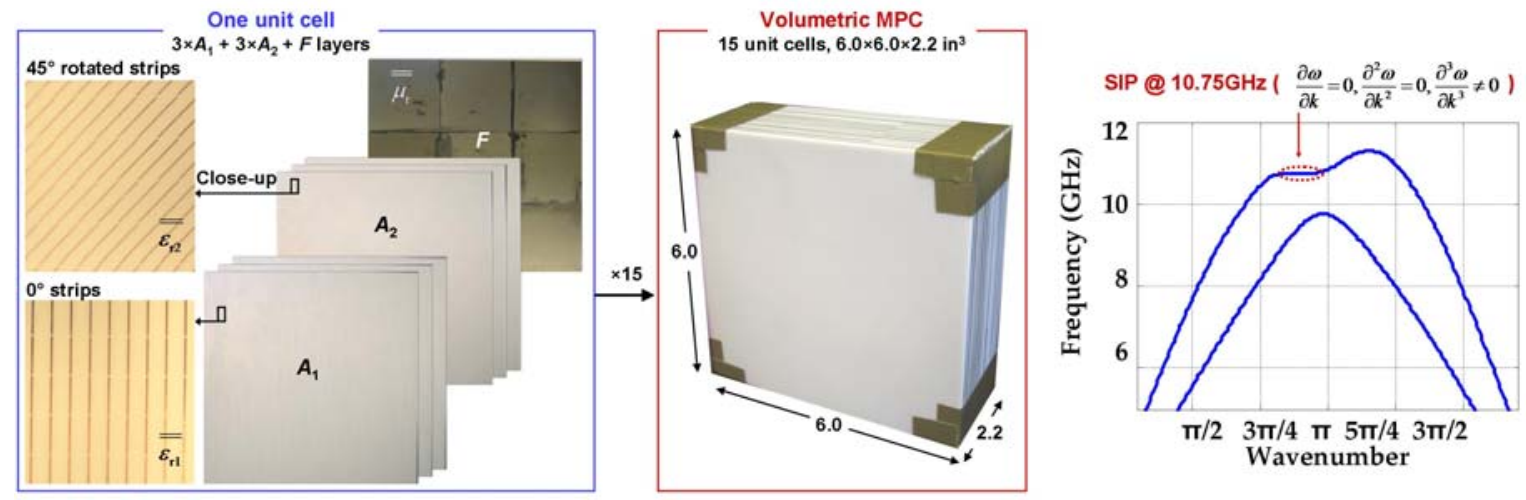

Fig. 2: 15 unit-cell volumetric MPC design and the its computed dispersion diagram.

Table 1: Material properties of dielectric and ferromagnetic layers in the volumetric MPC.

\begin{tabular}{|c|c|c|}
\hline Function & Materials & Material Properties \\
\hline A layer & Cu-strip printed Cuflon & $\begin{array}{c}\text { Cuflon: } \varepsilon_{\mathrm{r}}=2.05, \tan \delta=4.5 \times 10^{-4}, \text { thickness }=0.51 \mathrm{~mm} . \\
\text { Cu-strip: } 2.4 \mathrm{~mm} \times 0.13 \mathrm{~mm}\end{array}$ \\
\hline $\mathrm{F}$ layer & $\mathrm{Ca}, \mathrm{V}$-doped $\mathrm{Y}_{3} \mathrm{Fe}_{5} \mathrm{O}_{12}$ & $\varepsilon_{\mathrm{r}}=14.6,4 \pi M_{\mathrm{s}}=1600 \mathrm{G}, \Delta H=10 \mathrm{Oe}$, thickness $=0.64 \mathrm{~mm}$ \\
\hline
\end{tabular}

$* \varepsilon_{\mathrm{r}}$ is the dielectric constant, $\tan \delta$ is the dielectric loss tangent, $4 \pi M_{\mathrm{s}}$ is the saturation magnetization, $\Delta H$ is the ferromagnetic resonance linewidth.

Next, we tuned the dielectric/magnetic properties and geometrical dimensions of the MPC unit cell to achieve the stationary inflection behavior in the X-band $(\sim 11 \mathrm{GHz})$. The fine geometrical details of the printed Cuflon layers were modeled using the finite element method [6] for transmission/reflection computations. The optimized strip dimensions, including the $A$ and $F$ layer thickness, are listed in Table 1. Using the above parameters, an SIP was expected at $10.75 \mathrm{GHz}$ under a magnetic bias of $0.7 \mathrm{~T}$ (see Fig.2).

Figure 3(a) shows the vertically polarized transmittance of the MPC under an external magnetic bias of 0.7 Tesla. Coupling of the incoming wave into the MPC was best when the dielectric layers were oriented $45^{\circ}$ with respect to the vertical. As seen, the largest non-reciprocal transmission occurs very close to the design frequency at 11.85 GHz. Also shown in Fig. 3(b) is the calculated group delay associated with the frozen mode. Specifically, the forward wave took $7.22 \mathrm{~ns}$ to travel through the MPC. In contrast, the same distance in free-space is only $0.18 \mathrm{~ns}$, implying a 40 times slow down. 

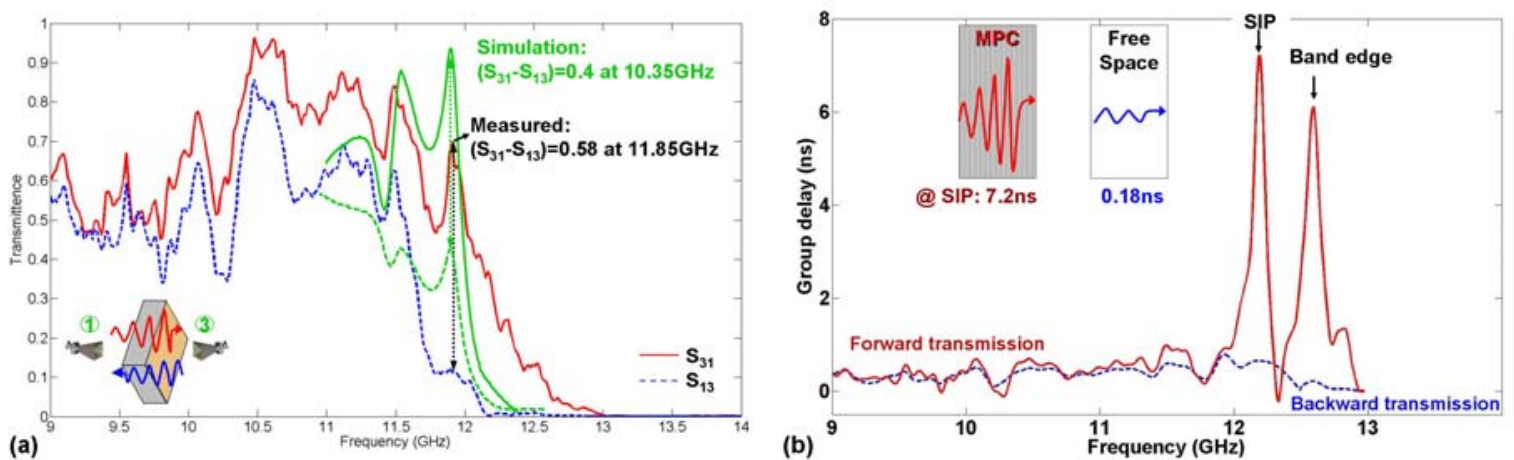

Fig. 3: (a) Measured and simulated non-reciprocal transmittance of the 15 unit-cell MPC structure. (b) Group delay calculated from the transmittance shown in (a).

\section{Experimental Demonstration MPC modes in Printed Structures}

As described in [8], we can also emulate the non-reciprocal MPC dispersion on simpler, printed periodic structures. Here, we designed and fabricated the 9-unit-cell shown in Fig. 4. The unit cell is a pair of partially coupled transmission lines printed on a ferrite substrate $\left(\mathrm{CVG}\right.$, calcium vanadium garnet, $4 \pi M_{\mathrm{s}}=976 \mathrm{G}, \Delta H=7 \mathrm{Oe}, \varepsilon_{\mathrm{r}}=14.12$ and $\tan \delta=10^{-4}$ ). The overall dimensions were $4.40 " \times 0.44 " \times 0.10^{\prime \prime}$.

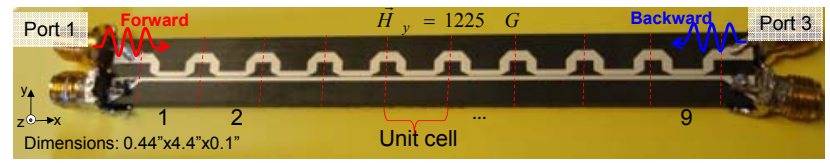

Fig. 4. Photograph of the actual printed transmission lines comprised of 9 unit-cells

As shown in Fig. 5, the printed unit cell is designed to exhibit the inflection behavior around $3 \mathrm{GHz}$. However, a direct measurement of the dispersion diagram is not possible due to the evanescent behavior near the feed ports. To remove the undesired contribution of the evanescent fields, we fabricated and measured a 9- unit-cell and an 8-unit-cell long printed structure. Doing so, the dispersion diagram of the printed MPC unit cell can be accurately evaluated using the transfer matrix approach [10]. Fig. 5(a) shows the corresponding measured and computed dispersion diagrams (computed curves were shifted down by $0.15 \mathrm{GHz}$ to coincide with measurements). It is seen that the spectral nonreciprocity and stationary inflection behavior is clearly observed in the measured data.

Unidirectional transmittance of the 9-unit-cell structure was also measured. As shown in Fig. 5(b), a pronounced contrast $\left(\left|\mathrm{S}_{13}\right|-\left|\mathrm{S}_{31}\right|=0.65\right)$ between forward and backward transmittances occurs around $2.9 \mathrm{GHz}$ due to the strong spectral asymmetry at that frequency.

$\kappa-\omega$ diagram

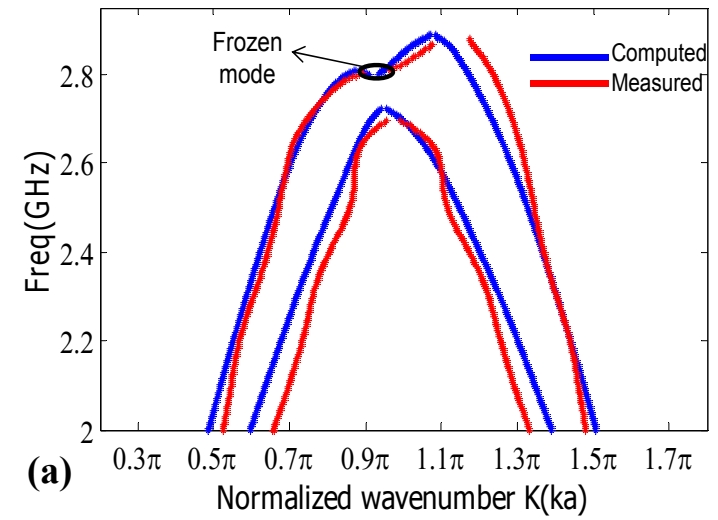

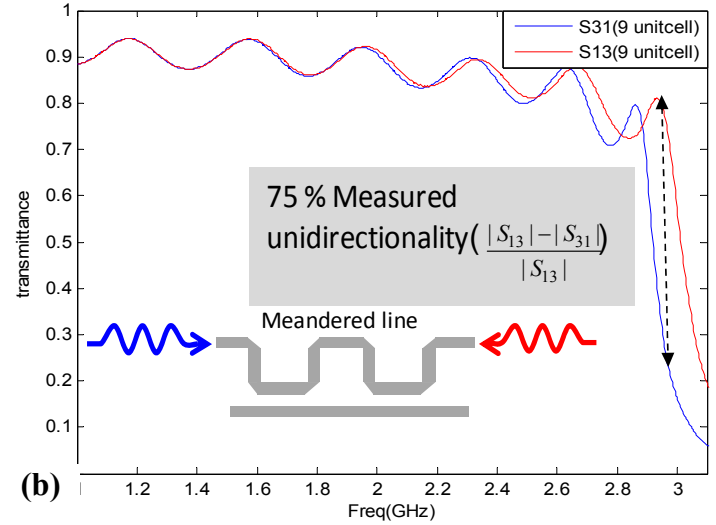

Fig. 5: (a) Measured and computed K- $\omega$ diagrams of the periodic printed transmission lines, (b) forward and backward transmittances for the 9 unit-cell structure. 


\section{Conclusions}

For the first time, we experimentally demonstrated the unique modes supported by magnetic photonic crystals. Spectral non-reciprocity, leading to unidirectional propagation and the existence frozen mode were validated in volumetric and printed structures. Specifically, a 15 unit-cell volumetric MPC prototype was constructed and found to support frozen mode at $11.85 \mathrm{GHz}$ with a $0.70 \mathrm{~T}$ external bias field. Correspondingly, the forward electromagnetic wave was slowed down by $\sim 40$ times with respect to the free space propagation. A validation of the printed MPC structure was also carried out, demonstrating the slow-wave support. Spectral asymmetry was demonstrated by directly calculating the dispersion diagram from measured data. With these experimental validations, the MPCs can provide new avenues for novel non-reciprocal, tunable and highly-sensitive electromagnetic devices.

\section{Acknowledgments}

This work was supported by Lockheed Martin Corporation under Grant Agreement/No. CDA09-034-PE.

\section{References}

1. C. Caloz, "Perspectives on EM metamaterials," Mater. Today, vol. 12, pp. 12-20, 2009.

2. H. Chen, B.I. Wu, J.A. Kong, "Review of electromagnetic theory in left-handed materials," J. Electromagnet. Wave., vol. 20, pp. 2137-2151, 2006.

3. Figotin and I. Vitebskiy, "Nonreciprocal magnetic photonic crystals," Phys. Rev. E, vol. 63:066609, 2001.

4. Figotin and I. Vitebskiy, "Electromagnetic unidirectionality in magnetic photonic crystals," Phys. Rev. B, vol. $67: 165210,2003$

5. G. Mumcu, K. Sertel, and J.L. Volakis, "Miniature antennas and arrays embedded within magnetic photonic crystals." IEEE Antenn. Wirel. Pr., vol. 5, pp. 168-171, 2006.

6. S. Yarga, K. Sertel, and J.L. Volakis, "Non-reciprocal radiation using magnetic photonic crystals," IEEE Antennas and Propagation Society Symposium, Charleston, SC, 2009.

7. J. Smit and H.P.J. Wijn, Ferrites, Philips Tech. Library, Eindhoven, 1959.

8. M.B. Stephanson, K. Sertel and J.L. Volakis, "Frozen modes in Coupled Microstrip Lines Printed on Ferromagnetic Substrates", IEEE Microwave Wireless Compon.Lett., vol. 18, pp. 305-307, 2008.

9. L. Zhang, S. Yarga, K. Sertel and J.L. Volakis, "Experimental validation of Non-reciprocal Properties Found in Volumetric Magnetic Photonic Crystals," Antennas and Propagation Society International Symposium, July 2010.

10. N. Apaydin, K. Sertel and J.L. Volakis, "Demonstration of Unidirectional Printed Structures Emulating Magnetic Photonic Crystals," Antennas and Propagation Society International Symposium, pp.1-4, July 2010. 